\title{
Best-Practices für die Gestaltung von IT-Service-Katalogen und den Einsatz von Self-Service-Portalen
}

\author{
Sebastian Floerecke (iD
}

Eingegangen: 29. September 2020 / Angenommen: 14. Dezember 2020 / Online publiziert: 23. Dezember 2020

(C) Der/die Autor(en) 2020, korrigierte Publikation 2021

Zusammenfassung Die Gestaltung von IT-Service-Katalogen stellt Unternehmen unterschiedlichster Branchen vielfach vor eine große Herausforderung. Denn ihnen mangelt es an bewährten, hinsichtlich ihrer Eignung evaluierten Vorlagen. Selbst Empfehlungen allgemeiner Art sind in der wissenschaftlichen Literatur nur vereinzelt zu finden. Vorliegender Beitrag greift diese Problemstellung auf und liefert auf Basis einer Einzelfallstudie innerhalb eines Großunternehmens unter Einbeziehung der vorhandenen Literatur - Forschungsarbeiten, Erfahrungsberichte aus der Praxis und Webauftritte von Tool-Herstellern - eine Zusammenstellung von Best-Practices, welche die Neuerstellung und Überarbeitung von IT-Service-Katalogen leiten können. Besonderes Augenmerk liegt auf dem in der Forschung bislang weitgehend außer Acht gelassenen Zusammenspiel mit Self-Service-Portalen. Damit können sich interne und externe Kunden über die angebotenen IT-Services informieren und verschiedenste Arten von Service-Requests wie Neubestellungen, Änderungen und Kündigungen initiieren. Unternehmensentscheider und IT-Verantwortliche erhalten mit diesem Beitrag eine Aufstellung an Kriterien und Anforderungen, die bei der Auswahl, der prozessualen und systemischen Integration sowie dem Customizing eines Self-Service-Portals von besonderer Bedeutung sind.

Schlüsselwörter IT-Service-Katalog $\cdot$ Self-Service-Portal $\cdot$ IT-ServiceManagement $\cdot$ Best-Practices $\cdot$ Einzelfallstudie $\cdot$ Literaturrecherche

\footnotetext{
S. Floerecke $(\square)$

Lehrstuhl für Wirtschaftsinformatik mit Schwerpunkt Informations- und IT-Service-Management, Universität Passau, Innstraße 43, 94032 Passau, Deutschland

E-Mail: sebastian.floerecke@uni-passau.de
} 


\title{
Best Practices for the Design of IT Service Catalogues and the Use of Self-Service Portals
}

\begin{abstract}
The creation of an IT service catalogue is a major challenge for many companies of different industries. There is a lack of proven and regarding their suitability evaluated templates. Even recommendations of general nature are scarce in the scientific literature. This paper addresses this problem and provides, based on a single-case study within a large company including the available literature (research studies, practical reports and websites of tool providers), a set of best practices, which can guide the creation and revision of IT service catalogues. Particular emphasis is placed on the interplay with self-service portals, which has been largely ignored by researchers to date. Self-service portals allow both internal and external customers to obtain information about the offered IT services and to initiate different types of service requests, such as orders, changes and terminations. Corporate decision-makers and IT managers get a list of criteria and requirements that are of decisive importance for the selection, processual and systemic integration as well as customizing of self-service portals.
\end{abstract}

Keywords IT Service Catalogue · Self-Service Portal · IT Service Management · Best Practices $\cdot$ Single-Case Study $\cdot$ Literature Research

\section{Motivation und Problemstellung}

Der IT-Service-Katalog eines Unternehmens ist eine Aufstellung, die transparent machen soll, welche IT-Services zu welchen Merkmalsparametern und zu welchem Preis von Mitarbeitern der eigenen (interne Kunden) und/oder einer anderen Organisation (externe Kunden) bezogen werden können (Macias und Alonso 2019). IT-Service-Kataloge schaffen eine gemeinsame Kommunikationsgrundlage zwischen Anbieter und Nachfrager und fungieren als Basis zahlreicher Prozesse des IT-ServiceManagements (ITSM) wie dem Request-, Incident- und Configuration-Management (Beims und Ziegenbein 2014) sowie von Teilen des betrieblichen Rechnungswesens (Grawe und Fähnrich 2008). Vor diesem Hintergrund kommt der zweckmäßigen und zielorientierten Gestaltung des IT-Service-Katalogs in der betrieblichen Praxis eine hohe Bedeutung zu. Da es allerdings an bewährten, hinsichtlich ihrer Eignung evaluierten Vorlagen mangelt und die wenigen vorhandenen für die direkte, praktische Umsetzung überwiegend unzureichend beschrieben sind, stellt dieses Vorhaben Unternehmen unterschiedlichster Branchen vielfach vor eine große Herausforderung (Nissen et al. 2014; Scholderer 2017; Sembiring und Surendro 2016). Auch die Information Technology Infrastructure Library (ITIL) - die führende Sammlung von Leitlinien, Schulungen und Zertifizierungsprogrammen für die ITSM-Branche bietet in dieser Hinsicht keine konkreten Gestaltungsempfehlungen (Axelos 2019). Selbst Empfehlungen allgemeiner Art liegen in der wissenschaftlichen Literatur nur vereinzelt (beispielsweise Macias und Alonso (2019); Schulz (2015)) vor. Eine Konsolidierung des Forschungsstands fehlt weitgehend (Macias und Alonso 2018). 
Unter diesen Umständen sind Praktiker bei der Gestaltung ihres IT-Service-Katalogs größtenteils auf sich allein gestellt.

\section{Ziele und Forschungsdesign}

Auf Basis eines Projekts zur grundlegenden Umgestaltung des vorhandenen ITService-Katalogs mit zahlreichen Workshops und Experteninterviews mit internen ITSM- und IT-Service-Katalog-Spezialisten sowie Herstellern und Dienstleistern von ITSM-Software-Suiten und Self-Service-Portalen wurden Best-Practices für die Gestaltung von IT-Service-Katalogen abgeleitet (erstes Ziel). Dazu wurden während der Datenerhebungen ausführliche handschriftliche Notizen angefertigt, die im Nachgang mittels qualitativer Inhaltsanalyse (Mayring 2010) ausgewertet wurden. Zusätzlich in die Untersuchung einbezogen wurden die Ergebnisse einer den Richtlinien von Webster und Watson (2002) folgenden strukturierten Literaturrecherche. Diese umfasste Forschungsarbeiten, Erfahrungsberichte aus der Praxis und Webauftritte aus dem Umfeld von ITSM-Software-Suiten und Self-Service-Portalen. Gesucht wurde bei Google (Webauftritte von Tool-Herstellern und Praktikerberichte) und in führenden wissenschaftlichen Datenbanken - ACM Digital Library, Google Scholar, IEEE Explorer, Science-Direct und SpringerLink. Als Suchwörter dienten „Service-Katalog“ in Verbindung mit „Aufbau“, „Design“, „Struktur“, „Vorlagen“, „Erfolgsfaktoren“ und „Best-Practices“ (wie auch ihre englischsprachigen Äquivalente). Die angewandte Forschungsmethodik entspricht insgesamt einer Einzelfallstudie (Yin 2018).

Der Schwerpunkt dieser Untersuchung lag auf dem Zusammenspiel von IT-Service-Katalogen und Self-Service-Portalen (zweites Ziel). In dieser Hinsicht hat sich in den vergangenen Jahren auf dem Markt ein breites Angebot an zumeist in ITSMSoftware-Suiten (Beispiele: Cherwell, EasyVista und ServiceNow) integrierten Modulen und vereinzelten Stand-Alone-Lösungen (Beispiel: Enterprise Service Catalog von PMG) entwickelt (Brooks und Curti 2014). Innerhalb des betrachteten Projekts wurde ein Self-Service-Portal ausgewählt, das in einem nachfolgenden Umsetzungsprojekt eingeführt werden soll.

Ein Self-Service-Portal ist eine Webanwendung, mit deren Hilfe sich interne und externe Kunden über die sich im IT-Service-Katalog befindlichen IT-Services informieren und verschiedene Arten von Service-Requests wie Neubestellungen, Änderungen und Kündigungen auslösen können - ohne mit einer Kontaktperson aus dem Unternehmen sprechen zu müssen (Brooks und Curti 2014). Im Mittelpunkt steht, Personen mit dem für ihre Tätigkeiten notwendigen IT-Equipment (Hardware und Software) auszustatten. Die IT wird von manuellen Routinearbeiten entlastet und gewinnt so freie Kapazitäten für wertschöpfende und strategisch wichtigere Aufgaben. Häufig resultiert daraus ein reduzierter Personalbedarf mit entsprechenden Einsparungsmöglichkeiten. Was die Auswahl, die Integration und die Verwendung von Self-Service-Portalen auf Basis von IT-Service-Katalogen anbelangt, ist die Forschung noch am Anfang (Jäntti 2013; Nord et al. 2016).

Analyseobjekt der Einzelfallstudie ist ein deutsches Unternehmen mit rund 10.000 Mitarbeitern, dessen IT-Service-Katalog über die Jahre auf etwa 1000 IT-Services 
anwuchs und damit für Kunden, aber auch für die IT selbst, zunehmend unübersichtlicher wurde. Bezieher der IT-Services sind überwiegend interne Kunden. Neben der kaum noch zu überblickenden Anzahl waren die Bezeichnungen und Beschreibungen der einzelnen IT-Services und ihre hierarchische Strukturierung für beide Seiten vielfach nur schwer verständlich. Die Folge waren vermehrte, zeitaufwendige Nachfragen per Telefon und E-Mail von Seiten der Kunden und einer damit einhergehenden allgemeinen niedrigen Kundenzufriedenheit. Unter diesen Umständen war eine grundlegende Umgestaltung des IT-Service-Katalogs in Einheit mit der Einführung eines Self-Service-Portals (Bestellungen bis dato hauptsächlich per E-Mail, teils per Telefon), auch in wegen der Corona-Pandemie wirtschaftlich angespannten Zeiten, aus Sicht der IT-Verantwortlichen unausweichlich.

\section{Die beiden Sichten eines IT-Service-Katalogs}

Ein IT-Service-Katalog ähnelt der Speisekarte eines Restaurants (Macias und Alonso 2018). Auf der obersten Kategorisierungsebene finden sich beispielsweise Vorspeisen, Hauptgerichte - eventuell verfeinert in die Unterkategorien Rind, Fisch und vegetarisch - Nachspeisen und Getränke. Darunter sind jeweils die einzelnen, bestellbaren Gerichte und Getränke platziert. Eine gänzlich andere Sicht auf eine Speisekarte ist die des Küchenpersonals. Deren Gedanken kreisen vornehmlich darum, welche Schritte sie in welcher Reihenfolge umsetzen müssen und welche Zutaten sie benötigen, um die entsprechenden Gerichte zubereiten und Getränke ausschenken zu können.

Übertragen auf den IT-Bereich eines Unternehmens: Es werden zwei Sichten auf den IT-Service-Katalog mit jeweils unterschiedlichen Informationen benötigt - eine interne IT-Sicht und eine Kundensicht (Axelos 2019; Beims und Ziegenbein 2014):

- Bei der internen IT-Sicht werden die IT-Services mit den zu ihrer jeweiligen Erbringung notwendigen technischen Details beschrieben. Es findet somit ein Mapping zwischen IT-Services und IT-Systemen/-Komponenten statt. Dazu dient eine Configuration-Management-Database (CMDB). Die interne IT-Sicht umfasst zudem die Definition von Genehmigungsprozessen und vom Kunden im Zuge der Beschaffung und Entsorgung von IT-Komponenten verborgene Aktivitäten.

- Bei der Kundensicht werden IT-Services in der Sprache der Kunden beschrieben. Dabei sollte so weit wie möglich auf technische Details verzichtet werden. Auf dieser Grundlage können Kunden einen Service-Request unterschiedlichster Art auslösen.

Studien zeigen, dass bei Weitem nicht allen Unternehmens- und IT-Verantwortlichen die Bedeutung beider Sichten und deren notwendigen engen Verzahnung bewusst ist und deshalb des Öfteren nur eine von beiden umgesetzt wird (Nord et al. 2016). In diesem Beitrag ist das Hauptaugenmerk auf die Kundensicht - das Schaufenster der IT - gerichtet. Die interne IT-Sicht ist Teil einer bereits geplanten Nachfolgestudie. 


\section{Ergebnisse der Einzelfallstudie}

In diesem Kapitel werden die Ergebnisse der durchgeführten Einzelfallstudie präsentiert: Zunächst werden Best-Practices hinsichtlich (1) des Gestaltungs- und Pflegeprozesses eines IT-Service-Katalogs im Allgemeinen, (2) der Strukturierungsebenen eines IT-Service-Katalogs sowie (3) der Definition und Beschreibung einzelner ITServices aufgeführt. Der Schwerpunkt liegt auf den darauffolgenden Kriterien und Anforderungen, die bei der Auswahl, der Integration und dem Customizing eines auf Basis eines IT-Service-Katalogs arbeitenden Self-Service-Portals von besonderer Bedeutung sind.

\subsection{Der Gestaltungs- und Pflegeprozess eines IT-Service-Katalogs im Allgemeinen}

Nachdem zahlreiche ITSM-Prozesse und die dafür verantwortlichen Abteilungen und Gruppen auf Grundlage des IT-Service-Katalogs agieren, ist es aus gesamtunternehmerischer Sicht unabdingbar, diese bei der Gestaltung aktiv einzubeziehen. Gleichermaßen müssen Vorschläge für den Aufbau des IT-Service-Katalogs fortlaufend mit repräsentativen Kunden getestet werden. Schließlich sind sie es, die später auf dessen Basis Service-Requests auslösen. Der Entwicklungsprozess muss also für alle Stakeholder transparent sein und es dürfen keine Entscheidungen von nur wenigen IT-Verantwortlichen im Alleingang getroffen werden. Eine agile Vorgehensweise ist dabei unerlässlich.

Wurden sämtliche IT-Services des Unternehmens gemeinschaftlich identifiziert und definiert, und steht die grundlegende Struktur des IT-Service-Katalogs, besteht die Aufgabe eines jeden Service-Owners darin, eine vollständige Beschreibung der von ihm verantworteten IT-Services zu erstellen. Der Katalog-Manager überprüft die einzelnen Beschreibungen, passt diese bei Bedarf in Rücksprache an und überträgt diese in den IT-Service-Katalog.

Der Katalog-Manager trägt im weiteren Verlauf die Verantwortung für die Pflege des IT-Service-Katalogs und sorgt dafür, dass sämtliche darin enthaltenen Informationen korrekt und auf dem aktuellen Stand sind. Zur eindeutigen Festlegung und Bekanntmachung der Prozesse und der Verantwortlichkeiten rund um den ITService-Katalog sollte eine IT-Service-Katalog-Policy verabschiedet werden.

\subsection{Die Strukturierungsebenen eines IT-Service-Katalogs}

IT-Service-Kataloge sollten aus einer flachen, zwei- bis maximal dreistufigen Hierarchie bestehen: Top-Level-Kategorie $>$ Sub-Kategorie $>$ Beziehbarer IT-Service (Beispiel: Mobiler Arbeitsplatz > Laptops > Laptop-Business-Klein). Jede Ebene (TopLevel-Kategorie, Sub-Kategorie, Beziehbarer IT-Service) sollte zur besseren Übersichtlichkeit und Auffindbarkeit nicht mehr als zehn Elemente enthalten. Diese Restriktion wird die allermeisten Unternehmen nicht einschränken, bietet es doch Platz für bis zu $1000(10 * 10 * 10)$ IT-Services.

Für die Top-Level-Kategorie eignet sich der Einsatzbereich, wie beispielsweise PC-Arbeitsplatz, Telefonie und Netzwerk \& Server. Von der Verwendung von 
Abteilungen oder Gruppen innerhalb von Kategorienbezeichnungen wird abgeraten. Interne, aber vor allem externe Kunden kommen damit, wie die Ergebnisse dieser Fallstudie zeigen, schlecht zurecht. Einer jeden Top-Level-Kategorie sind eine oder mehrere Sub-Kategorien zuzuordnen, die einen oder mehrere beziehbare IT-Services fachlich aussagekräftig zusammenfassen. Für IT-Services, die sich nur schwer in Top-Level- und Sub-Kategorien einordnen lassen, bietet sich jeweils eine Kategorie Rest oder Weitere an. Die für Kunden auswählbaren IT-Services befinden sich auf der dritten Ebene. Bei Unternehmen mit einem vergleichsweise überschaubaren IT-Leistungsportfolio kann die mittlere Ebene (Sub-Kategorie) entfallen. Grundsätzlich wird nicht nur eine Beschreibung einzelner IT-Services (siehe Abschn. 4.3), sondern einer jeden vorhandenen Kategorie benötigt. So können Kunden direkt erkennen, was sich jeweils dahinter verbirgt.

\subsection{Definition und Beschreibung einzelner IT-Services}

Beziehbare IT-Services der Kundensicht sollten wie IT-Services, und eben nicht vordergründig wie Produkte und Applikationen (technische Perspektive), bezeichnet und beschrieben werden. Aus der Kundenperspektive stellt der Nutzen - das Was, nicht das Wie - das entscheidende Kriterium dar. Dazu ein anschauliches Beispiel: Beim beziehbaren IT-Service Video-Konferenz können die zur Auswahl stehenden Applikationen, wie etwa Skype und Webex, als Option gewählt werden. Die Anwendungen Skype und Webex stehen für sich allein genommen für keinen IT-Service. Denn dahinter verbergen sich noch eine Vielzahl von Zusatzleistungen wie Support und Wartung und technischen Komponenten wie Server und Netzwerk.

Tab. 1 Notwendige Attribute einer IT-Service-Beschreibung

\begin{tabular}{l}
\hline IT-Service-Beschreibung \\
\hline Bezeichnung \\
Versionsnummer \\
Kurzbeschreibung \\
Hersteller \\
Nutzerhandbücher und technische Informationen \\
Bilder und Videos \\
Gewicht und Maße (bei Hardware) \\
Vertragslaufzeit \\
SLAs \\
Preis (monatlicher Preis/Einmalpreis) \\
Service-Owner \\
Zu kontaktierende Person bei Rückfragen \\
Durchschnittliche Dauer bis zur Service-Bereitstellung \\
Wartungsfenster \\
Benötigtes Endgerät zur Nutzung \\
Ähnliche IT-Services \\
Datum letzter Aktualisierung und Version der Beschreibung
\end{tabular}


Neben atomaren IT-Services sollten, wann immer möglich, sogenannte ServiceBundles, bestehend aus einzelnen IT-Services, geschaffen werden. Dieser Ansatz erleichtert Kunden die Bestellung von technisch kompatiblen und fachlich sinnvollen Kombinationen von IT-Services. Ein typisches Beispiel eines Service-Bundles ist ein kompletter Büro-Arbeitsplatz (PC, Standard-Office-Software, Spezial-Software, Telefon, etc.). Werden IT-Services auch externen Kunden angeboten, lässt es sich damit von Wettbewerbern, deren Portfolios keine derartigen Service-Bundles aufweisen, effektiv differenzieren.

Die einzelnen IT-Services müssen mittels relevanter Attribute beschrieben werden. Dazu zählen, ohne Anspruch auf Vollständigkeit zu erheben, insbesondere die in Tab. 1 abgebildeten.

Da Besteller mitunter über wenig Kenntnis verfügen, welcher Preis im Unternehmensumfeld hoch beziehungsweise niedrig ist, kann es hilfreich sein, zusätzlich zum tatsächlichen Preis grundlegende Preiskategorien (Beispiel: günstig, mittel und teuer) zur Orientierung einzuführen. Wird ein IT-Service gleichzeitig in verschiedenen Service-Level-Agreement (SLA)-Varianten (Beispiel: Gold, Silber und BestEffort) angeboten, sollten diese keinesfalls als gänzlich eigene IT-Services, sondern als einer mit für Kunden unterschiedlichen, wählbaren Optionen geführt werden. Dieses Vorgehen würde ansonsten zu einem unnötigen Aufblähen des IT-ServiceKatalogs führen. Die Möglichkeit der Service-Individualisierung für Kunden gilt es auf ein Minimum zu beschränken, da dies Risiken und Kosten erheblich erhöhen würde.

\subsection{IT-Service-Kataloge im Zusammenspiel mit Self-Service-Portalen}

Grundsätzliches Die Bedienung von Self-Service-Portalen muss selbsterklärend sein. Denn Nutzer geben erfahrungsgemäß durchschnittlich nur zwei- bis dreimal jährlich eine Bestellung auf Basis des IT-Service-Katalogs auf. Kunden müssen also in der Lage sein, das Self-Service-Portal zu bedienen, ohne zuvor eine Schulung zu besuchen. Aufgrund des im unternehmerischen Umfeld spürbaren und voraussichtlich durch die Corona-Pandemie weiter zunehmenden Trends hin zu mobilen Endgeräten sollte zusätzlich zur Desktop-Version eine mobile Version in Erwägung gezogen werden.

ITSM-Self-Service-Portal vs. E-Commerce-System Ein Self-Service-Portal unterscheidet sich grundlegend von einem E-Commerce-System: Bei einem Self-Service-Portal im ITSM-Kontext geht es nicht vordergründig darum, eine Kaufentscheidung zu induzieren. Vielmehr sollen Mitarbeiter, ob innerhalb der eigenen oder einer anderen Organisation, mit dem für ihre Tätigkeiten notwendigen IT-Equipment ausgestattet und mittels IT-Services bestmöglich unterstützt werden. Deshalb spielen hier Cross-Selling-Maßnahmen eine untergeordnete Rolle. Ein Self-Service-Portal zielt auf das Anbieten und das Bereitstellen von IT-Services, und eben nicht auf den Verkauf von Produkten, ab. Services und Produkte unterscheiden sich im Lebenszyklus, den zur Erbringung notwendigen Aktivitäten und den Eigentumsverhältnissen wesentlich voneinander. Aus diesem Grund sind generischen Shop-Systemen die zentralen ITSM-Konstrukte wie Configuration-Items, Incidents und SLAs fremd. 
Dieser Umstand erschwert deren Erweiterung über die reine Bestellauslösung in Richtung zusätzlicher ITSM-Prozesse erheblich. Von diesem Vorhaben raten selbst die führenden Anbieter von E-Commerce-Systemen ab. Zur Realisierung eines zentralen Self-Service-Portals der IT eignen sich generische Shop-Systeme folglich nicht.

Neben Cross-Selling-Maßnahmen ist auch der tatsächliche Lagerbestand, verglichen mit E-Commerce-Systemen, im Self-Service-Portal-Umfeld nicht in dem Maße von Bedeutung. Es empfiehlt sich, auf die doch meist aufwendige Integration eines Self-Service-Portals mit dem Warenwirtschaftssystem zu verzichten. Viel entscheidender ist der systemseitige Hinweis auf technische Konflikte zwischen aktuell bezogenen und gerade ausgewählten IT-Services (Beispiel: Abhängigkeit zwischen CAD-Software und notwendiger Hardware) und die Anzeige von jeweils zur Nutzung zusätzlich erforderlichen IT-Services (Beispiel: Zur Benutzung einer App wird ein Smartphone oder Tablet benötigt).

IT-Service-Katalog vs. IT-Service-Portfolio In ein Self-Service-Portal darf nur der IT-Service-Katalog, nicht das komplette IT-Service-Portfolio, integriert werden - also keine IT-Services, die aktuell zwar seitens Kunden vereinzelt bezogen werden, aber nicht neu vertrieben werden sollen sowie IT-Services, die sich noch in der Entwicklungsphase befinden. Werden im Unternehmen Cloud-Computing-Services verwendet, sollten diese (unabhängig, ob intern oder extern gehostet und erbracht) gleichermaßen in den IT-Service-Katalog und das Self-Service-Portal aufgenommen werden. So wird entgegengewirkt, dass einzelne Personen oder Abteilungen CloudComputing-Services ohne Rücksprache mit der IT-Abteilung bestellen - die Basis für das Entstehen einer Schatten-IT mit all ihren negativen Folgen.

Berechtigungen, Kundenspezifität und Robustheit In der Regel ist nicht jeder interne und externe Kunde berechtigt, sämtliche IT-Services im Allgemeinen und in allen SLA-Varianten im Speziellen zu beziehen. Auf keinen Fall sollten Kunden im Self-Service-Portal IT-Services angezeigt bekommen, die sie schlussendlich nicht bestellen können. Diese Vorgabe lässt sich mittels eines Rollenmodells umsetzen. Was die Preise betrifft, müssen die kundenindividuellen Preise (häufig unterschiedliche Rabatte bei externen Kunden) herangezogen werden können. Ein Self-ServicePortal muss darüber hinaus robust gegenüber fehlerhaften Benutzereingaben sein. Ein Beispiel hierfür ist die Angabe einer nichtexistierenden Kostenstelle. Bei einer Bestellung durch den Kunden sollten daher entsprechende Felder im Self-ServicePortal weitestmöglich mit Inhalt vorbelegt sein. Dazu müssen Benutzerinformationen mit kaufmännischen und organisatorischen Informationen angereichert werden.

Erleichterung der Identifizierung von IT-Services Im Self-Service-Portal kann es durchaus sinnvoll sein, bestimmte IT-Services gleichzeitig mehreren Kategorien zuzuordnen. Denn Kunden sollen einen IT-Service einfach finden können, gegebenenfalls über mehrere Wege. Wichtig ist hierbei, dass jeder IT-Service zur Vermeidung von Redundanz intern nur einmal angelegt wird - lediglich die Zuordnung zu Kategorien kann mehrfach erfolgen. Andererseits sollten im Self-Service-Portal enthaltene IT-Services, die nie oder nur sehr selten bestellt werden, nach einem 
bestimmten Zeitraum wieder entfernt (wahlweise nur aus dem Self-Service-Portal oder vollständig aus dem IT-Service-Katalog) werden.

Neben der Kategorienstruktur empfiehlt sich eine alphabetische Auflistung der IT-Services. Die gefragtesten und neuesten IT-Services sollten zusätzlich jeweils in einem separaten Bereich angezeigt werden. Ferner muss dem Kunden eine Suchfunktion zur Verfügung gestellt werden. Dazu ist es notwendig, gemeinsam mit Kunden entsprechende Suchwörter, sogenannte Tags, je bestellbarem IT-Service zu definieren. Lediglich die Einbeziehung der Bezeichnung und der Beschreibung reicht in der Regel nicht aus. Die Suche sollte um eine Autovervollständigungsfunktion ergänzt werden. Die Suchtreffer sollten seitens des Kunden nach unterschiedlichen Kriterien, wie etwa Alphabet, Datum der Aufnahme in den IT-Service-Katalog und Preis, sortiert werden können. Dieses Bündel an Maßnahmen erleichtert Kunden die Identifizierung für sie geeigneter IT-Services. Abgesehen davon muss der Kunde im Self-Service-Portal die Möglichkeit erhalten, den kompletten IT-Service-Katalog als PDF-Dokument downloaden zu können.

Für den Fall, dass ein Nutzer einen im Self-Service-Portal nicht enthaltenen ITService sucht, eignen sich sogenannte Joker-Services. Dabei handelt es sich um ein leeres Service-Template, das der Kunde mit Wunschinformationen befüllen kann. Die IT kann im Nachgang prüfen, ob sie in Zukunft einen derartigen IT-Service anbieten kann und auch möchte. Der wesentliche Vorteil eines Joker-Services gegenüber einer formlosen E-Mail und einer telefonischen Anfrage: Die IT erhält durch die Vorgabe von Pflichtfeldern von Beginn an einen relativ detailliert definierten ITBedarf.

Warenkorbfunktion und Favoritenliste Zusätzlich zu einer im Self-Service-Portal zwingend notwendigen Warenkorbfunktionalität sollte der Kunde die Möglichkeit erhalten, eine sogenannte Favoritenliste anzulegen und zu bearbeiten. In diese Liste kann der Kunde IT-Services, an denen er Interesse hat und gegebenenfalls in Zukunft bestellen möchte, als Merker abspeichern. Der Kunde muss die sich in der Favoritenliste befindlichen IT-Services bei Bedarf einzeln in den Warenkorb verschieben können. Hintergrund: Einige Kunden bestellen in regelmäßigen Abständen dieselben IT-Services (beispielsweise durch Assistenten/innen im Rahmen des OnboardingProzesses). Insbesondere für diese Personengruppe bedeutet eine Favoritenliste eine deutliche Erleichterung für den Auswahl- und Bestellvorgang.

Abzudeckende Use-Cases Neben der Neubestellung von IT-Services ist das Einbinden weiterer Use-Cases beziehungsweise Typen von Service-Requests in ein SelfService-Portal, wie Abb. 1 zeigt, entscheidend. Zur Wahrung der Übersichtlichkeit wurde auf die Assoziationen im Use-Case-Diagramm verzichtet. Akteure sind sowohl interne als auch externe Kunden.

Gesamtübersichten für Führungskräfte Ein Self-Service-Portal muss Führungskräften und deren Assistenten/innen die Möglichkeit bieten, die aktuell bezogenen IT-Services von jedem direkt zugeordneten Mitarbeiter in einer Gesamtübersicht einsehen zu können. Als Bezugsgröße bietet sich die jeweilige Gruppen- oder Ab- 


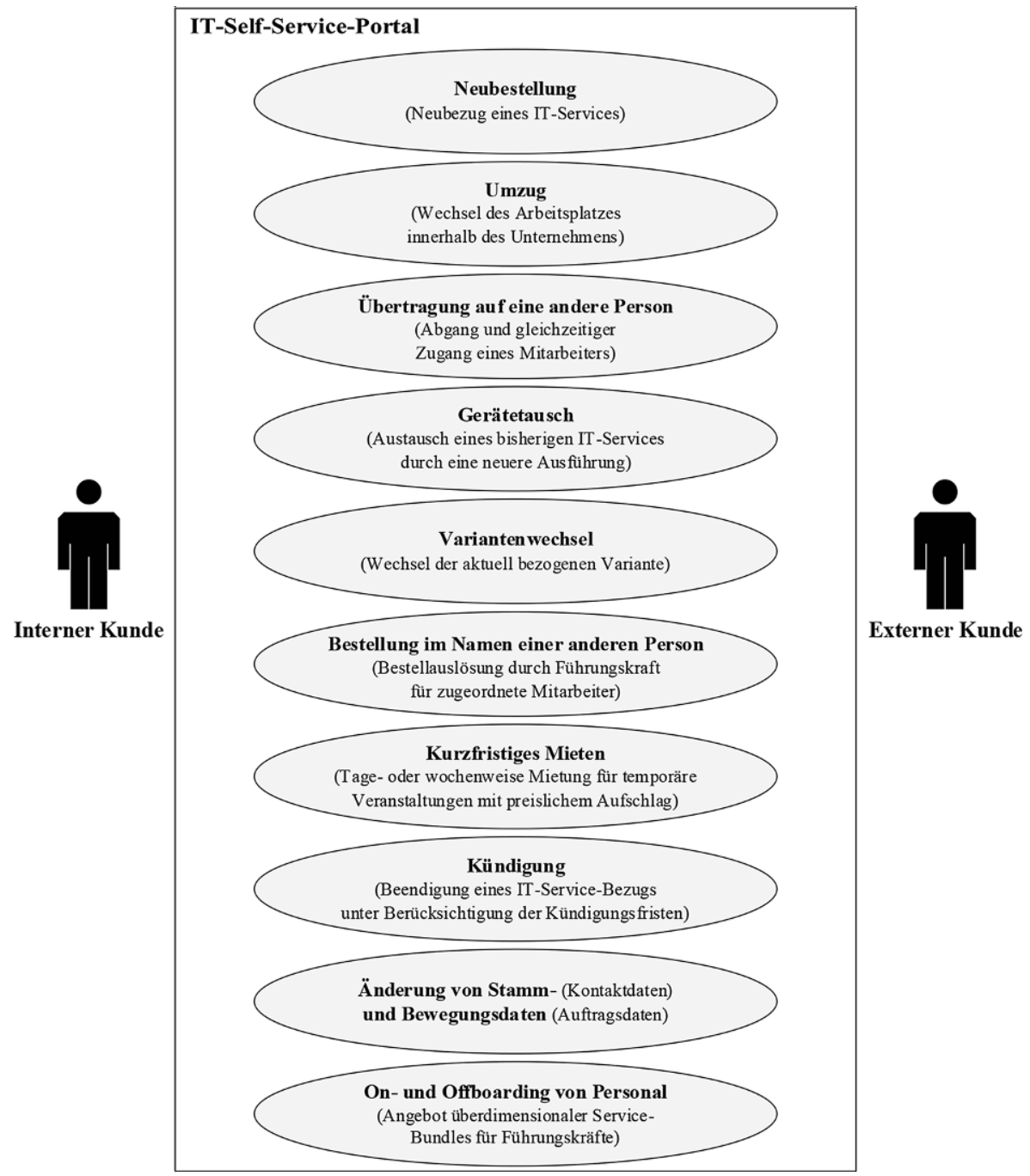

Abb. 1 In einem Self-Service-Portal abzudeckende Use-Cases

teilungskostenstelle an. Eine solche Gesamtübersicht dient neben der reinen Informationsfunktion insbesondere dem Offboarding-Prozess von Mitarbeitern.

Einbindung weiterer ITSM-Prozesse Neben den Requests rund um Standard-ITServices bietet es sich an, Requests von Dokumenten wie Unternehmensrichtlinien und Prozessbeschreibungen zu ermöglichen. Das Rollen- und Rechtemanagement - Anlegung eines Nutzers und Zuweisung spezifischer Berechtigungen innerhalb eines bestimmten Systems - muss in das Self-Service-Portal eingebunden werden. Selbiges gilt für das Incident-Management. Besonders zweckdienlich ist es, wenn der Kunde im Falle einer Störung auf Basis seiner Leistungsübersicht IT-Service-spezi- 
fische Incidents erstellen und verfolgen kann. Weitere vielversprechende Kandidaten für einzubindende ITSM-Prozesse sind insbesondere das Configuration- (Übersichten von Configuration-Items) und das Demand-Management (Erstellen und Verfolgen von Demands). Nach und nach soll somit ein zentrales Self-Service-Portal für möglichst viele IT-Belange der internen und externen Kunden entstehen.

Statuskonzept Nach der Auslösung eines Service-Requests erwarten Kunden eine aussagekräftige Statusanzeige im Self-Service-Portal. Dadurch lässt sich die Zahl schriftlicher und telefonischer Nachfragen reduzieren. Natürlich muss sichergestellt sein, dass der Prozess mit den Statusübergängen innerhalb der IT gelebt wird.

Automatisierte Beantwortung von Service-Requests Beginnend mit einem Service-Request sollte ein Workflow im ITSM-Kernsystem angestoßen werden. Ein solcher Workflow legt fest, welche Aktivitäten einzelne IT-Systeme und Personen in welcher Reihenfolge durchführen müssen, damit der jeweilige Use-Case (siehe Abb. 1) umgesetzt und abgerechnet wird. Hierbei ist auf einen möglichst hohen Automatisierungsgrad zu achten. Oberstes Ziel sollte ein möglichst durchgängiger, System- und Medienbrüche vermeidender Prozess ausgehend vom Service-Request, über die Umsetzung bis zur Abrechnung sein. Grundvoraussetzung ist ein hohes $\mathrm{Maß}$ an Integration von organisatorischen, kaufmännischen und technischen Software-Tools.

Genehmigungsworkflows Für sämtliche Use-Cases müssen spezifische Genehmigungsworkflows hinterlegt werden können. Ein Service-Request sollte erst dann bearbeitet werden, wenn die erforderliche Genehmigung seitens der dafür zuständigen Führungskraft vorliegt. Im Gegensatz zu den internen Kunden empfiehlt es sich bei externen Kunden, dass nur diejenigen Personen Zugang zum Self-ServicePortal erhalten, die gänzlich frei von Genehmigungen bestellen können. Zu jedem Genehmiger sollte ein Vertreter hinterlegt werden können.

\section{Schlussfolgerungen}

Mit diesem Beitrag erhalten Unternehmensentscheider und IT-Verantwortliche eine Zusammenstellung von Best-Practices für die Neuerstellung und die Überarbeitung von IT-Service-Katalogen. Diese auf die Kundensicht fokussierenden Aspekte können konkrete Gestaltungsvorhaben in Unternehmen leiten. Ein IT-Service-Katalog muss auf das jeweilige Unternehmen individuell zugeschnitten werden.

Bei einer Einzelfallstudie kann - trotz der Erweiterung um die vorhandene Literatur - kein Anspruch auf Vollständigkeit erhoben werden. Weitere Fallstudien in Unternehmen mit unterschiedlichen Kundensegmenten (interne vs. externe Kunden) und Größen sowie aus verschiedenen Branchen (auch der Vergleich privater vs. öffentlicher Sektor) und Ländern sind notwendig. Erste Studien zu den Spezifika kleiner und mittlerer Unternehmen liegen bereits vor (Arcilla et al. 2013).

Vorschläge für IT-Service-Kataloge müssen einer rigorosen Evaluation innerhalb realer Umgebungen unterzogen werden. Dieser Schritt wurde bislang vielfach un- 
terlassen (Macias und Alonso 2018). Wenngleich es keinen universalen IT-ServiceKatalog geben kann, sind für Praktiker branchenspezifische Referenz-IT-ServiceKataloge von großem Nutzen. Diese existieren jedoch bis dato nur für wenige Branchen wie beispielsweise dem Gesundheitswesen (Gerber et al. 2019).

Der Schwerpunkt dieser Untersuchung lag auf der Identifizierung der Kriterien und Anforderungen, die bei der Auswahl, der prozessualen und systemischen Integration sowie dem Customizing eines auf Basis eines IT-Service-Katalogs arbeitenden Self-Service-Portals von entscheidender Bedeutung sind. In dieser Hinsicht befindet sich die Forschung noch in den Anfängen. Vorliegender Beitrag trägt somit dazu bei, die Forschungslücke zu reduzieren.

Weiterhin fehlt es an Studien, die Unternehmensentscheider und IT-Verantwortliche dabei unterstützen, aus dem großen, unübersichtlichen Marktangebot an SelfService-Portalen das für die jeweiligen Anforderungen am besten geeignete zu wählen. Die vereinzelt vorliegenden Marktstudien von Gartner, Forrester und Co. (beispielweise Brooks und Curti (2014)) und eine kürzlich erschienene Evaluationsstudie zu einer spezifischen ITSM-Software-Suite (Schneider et al. 2019) bieten hierfür zwar eine erste Orientierung, aber keine ausreichende Unterstützung. Natürlich wird sich mit keinem der auf dem Markt erhältlichen Self-Service-Portale die darin gesetzten Erwartungen erfüllen lassen, wenn der zugrunde liegende ITService-Katalog nicht eine gewisse Mindestqualität aufweist.

Als Gesamtfazit lässt sich festhalten: Der Erfolg eines Self-Service-Portals hängt einerseits von den abgedeckten Service-Request-Typen (Use-Cases) und ITSM-Prozessen, der Datenqualität und -quantität, dem Integrationsgrad der IT-Landschaft und der Benutzerfreundlichkeit, andererseits vom aktiven Leben der Prozesse innerhalb der IT ab. Nur wenn all diese Faktoren zusammenkommen, wird sich der erhoffte Erfolg einstellen.

Funding Open Access funding enabled and organized by Projekt DEAL.

Open Access Dieser Artikel wird unter der Creative Commons Namensnennung 4.0 International Lizenz veröffentlicht, welche die Nutzung, Vervielfältigung, Bearbeitung, Verbreitung und Wiedergabe in jeglichem Medium und Format erlaubt, sofern Sie den/die ursprünglichen Autor(en) und die Quelle ordnungsgemäß nennen, einen Link zur Creative Commons Lizenz beifügen und angeben, ob Änderungen vorgenommen wurden.

Die in diesem Artikel enthaltenen Bilder und sonstiges Drittmaterial unterliegen ebenfalls der genannten Creative Commons Lizenz, sofern sich aus der Abbildungslegende nichts anderes ergibt. Sofern das betreffende Material nicht unter der genannten Creative Commons Lizenz steht und die betreffende Handlung nicht nach gesetzlichen Vorschriften erlaubt ist, ist für die oben aufgeführten Weiterverwendungen des Materials die Einwilligung des jeweiligen Rechteinhabers einzuholen.

Weitere Details zur Lizenz entnehmen Sie bitte der Lizenzinformation auf http://creativecommons.org/ licenses/by/4.0/deed.de.

\section{Literatur}

Arcilla M, Calvo-Manzano J, San Feliu T (2013) Building an IT service catalog in a small company as the main input for the IT financial management. Comput Stand Interfaces 36(1):42-53

Axelos (2019) ITIL Foundation: ITIL 4 edition (english edition) Bd. 4. TSO, Norwich 
Beims M, Ziegenbein M (2014) IT-Service-Management in der Praxis mit ITIL, 4. Aufl. Carl Hanser, München

Brooks JM, Curti D (2014) Critical capabilities for IT service catalog tools. Gartner, Stamford

Gerber N, Stuber K, Baacke L (2019) Referenzkatalog der ICT Services im Gesundheitswesen: Modell für ICT Service Management, Controlling und Benchmarking, 1. Aufl. ZHAW Zürcher Hochschule für Angewandte Wissenschaften, Wädenswil

Grawe T, Fähnrich KP (2008) Service Engineering bei IT-Dienstleistern. In: Fähnrich K-P, van Husen C (Hrsg) Entwicklung IT-basierter Dienstleistungen. Co-Design von Software und Services mit ServCASE, 1. Aufl. Physica-Verlag, Heidelberg, S 281-301

Jäntti M (2013) Exploring self-service support methods in IT service management. 10. International Conference on Service Systems and Service Management, Hong Kong

Macias CM, Alonso IA (2018) Review of proposals for the construction and management of the catalog of information technology services. IEEE Access 6:45335-45346

Macias CM, Alonso IA (2019) Proposal for the identification of information technology services in public organizations. Symmetry 11(10):1-32

Mayring P (2010) Qualitative Inhaltsanalyse: Grundlagen und Techniken, 12. Aufl. Beltz, Weinheim

Nissen V, Petsch M, Jung D, Praeg CP (2014) Empfehlungen für eine generelle IT-Service-Katalog-Struktur. In: Thomas O, Nüttgens M (Hrsg) Dienstleistungsmodellierung 2014, 1. Aufl. Gabler, Wiesbaden, S $133-154$

Nord F, Dörbecker R, Böhmann T (2016) Structure, content and use of IT service catalogs - Empirical analysis and development of a maturity model. 49. Hawaii International Conference on System Sciences, Manoa, Hawaii

Schneider D, Plate F, Auth G (2019) Evaluation von ITSM-Tools für Integration und Management von Cloud-Diensten am Beispiel von ServiceNow. 14. International Conference on Wirtschaftsinformatik, Siegen

Scholderer R (2017) IT-Servicekatalog: Services in der IT professionell designen und erfolgreich implementieren, 1. Aufl. dpunkt, Heidelberg

Schulz C (2015) IT-Katalogmanagement: Steuerungsinstrument für den internen Hard- und Softwarebestand. OBJEKTspektrum 3:68-72

Sembiring M, Surendro K (2016) Service catalogue implementation model. 4. International Conference on Information and Communication Technology, Neu-Delhi

Webster J, Watson RT (2002) Analyzing the past to prepare for the future: writing a literature review. Manag Inf Syst Q 26(2):13-26

Yin RK (2018) Case study research: design and methods, 6. Aufl. SAGE, Thousand Oaks 